\title{
Optimal Design of Sparse Reconfigurable Arrays Based on Multiple Measurement Vector Model
}

\author{
Haiou Shen ${ }^{+}$and Buhong Wang \\ Information and Navigation College, Air Force Engineering University, Xi'an, China
}

\begin{abstract}
In this paper, an innovative approach is presented for the optimal design of sparse reconfigurable antenna arrays. The synthesis of sparse arrays is recast as a simultaneous sparse approximation problem and modeled with multiple measurement vector (MMV). A multiple response extension of the sparse Bayesian learning (SBL) named M-SBL is exploited to solve the above optimization problem. The common element positions and individual element excitations for multiple radiation patterns are obtained simultaneously by searching out multiple sparse weight vectors. The proposed approach can dynamically reconfigure different patterns with a higher matching accuracy and array sparseness. A representative experiment is provided to validate the effectiveness and advantages of the proposed method.
\end{abstract}

Keywords: multiple measurement vector, simultaneous sparse approximation, pattern reconfigurable antenna, sparse antenna arrays, array synthesis

\section{Introduction}

Reconfigurable antenna arrays are able to radiate more than one pattern allows replacing a traditional multi-antenna and multi-task system by a single antenna system that is able to accomplish all these tasks, thus providing perfect performance advantages in reducing hardware complexity and saving space. It has many applications in communications, radars, remote sensing and biomedical imaging because of the realtime need to generate different patterns in a limited space [1-4]. Several methods have been proposed in the literatures for the optimal design of reconfigurable antenna arrays [5-8]. Reference [5] proposed a method of generating phase-only multiple pattern antenna arrays using particle swarm optimization (PSO) algorithm. Reference [6] achieved a pattern reconfigurable antenna array with uniform arrangement using common array aperture with continuously controllable feed network. The alternating projections technique [7] and the modified Woodward-Lawson technique [8] have also been presented to solve the above design problem. However, these synthesis techniques only concern with uniformly spaced multiple-pattern linear arrays, and thus require a large number of antenna elements spaced by $\lambda / 2$ to radiate the desired patterns when dealing with a large aperture. Naturally, non-uniform arrays have many advantages over uniform arrangements such as increased resolution, less mutual coupling effects and low cost, which are more preferable for some situations where the weight, the size, and the cost of antennas are limited.

Therefore, this paper is aimed at proposing an effective method for the optimal design of sparse reconfigurable arrays capable of radiating more than one desired patterns, which is a tough problem that can be equivalent to finding the minimum number of antenna elements and determining a common position distribution as well as one or multiple excitation vectors for the desired patterns with a small tolerance. More recently, the model, named nultiple measurement vector [9], enlightens us on a better solution for the synthesis of reconfigurable antenna with a sparse array layout. Based on the MMV model, an effective approach called M-SBL is presented here for the optimal design of sparse pattern reconfigurable antenna

\footnotetext{
+ Corresponding author. Tel.: + 13468822323.

E-mail address: 326519166@qq.com.
} 
arrays. The outline of this paper is organized as follows. The mathematical formulation for the proposed synthesis method is introduced in Section 2. The numerical analysis and assessment are presented in Section 3. Finally, the main conclusions are drawn in Section 4.

\section{Mathematical Formulation}

Assume that a linear array is composed of $N$ candidate antenna elements and $M$ different operative modes of a pattern reconfigurable array can be generated by varying the element excitations (include phases and amplitudes). The far-field array factor can be described as

$$
F^{(m)}(u)=\sum_{n=1}^{N} w_{n}^{(m)} \exp \left(\mathrm{j} k_{0} d_{n} u\right) \quad m=1, \ldots, M
$$

where $\mathrm{j}=\sqrt{-1}, k_{0}=2 \pi / \lambda$ is the spatial wavenumber and $\lambda$ is the wavelength. $u=\sin \theta$ and $\theta$ is the steering angle with respect to the linear array geometry. $w_{n}^{(m)}$ is the excitation coefficient of the $n$th element associated with the $m$ th pattern. $d_{n}=-(L / 2)+\Delta d(n-1)$ is the location of the $n$th candidate antenna elements with equal interelement spacing $\Delta d=L /(N-1)$.

Through discrete sampling at different $K(K \ll N)$ observation angle within the angular range [-1, 1], the sparse matrix form can be expressed as following

$$
\boldsymbol{F}^{(m)}=\boldsymbol{\Phi} \boldsymbol{w}^{(m)} \quad m=1, \ldots, M
$$

where $\boldsymbol{F}^{(m)}=\left[F^{(m)}\left(u_{1}\right), F^{(m)}\left(u_{2}\right), \ldots, F^{(m)}\left(u_{K}\right)\right]^{\mathrm{T}} \in \mathbb{C}^{K}$ is a sample vector of the $m$ th pattern at different $K$ observation angle, $\boldsymbol{w}^{(m)}=\left[w_{1}^{(m)}, w_{2}^{(m)}, \ldots, w_{N}^{(m)}\right]^{\mathrm{T}} \in \mathbb{C}^{N}$ is a excitation vector associated with the $m$ th pattern, and the observation matrix $\boldsymbol{\Phi}$ can be written as

$$
\boldsymbol{\Phi}=\left[\begin{array}{cccc}
\exp \left(\mathrm{j} k_{0} d_{1} u_{1}\right) & \exp \left(\mathrm{j} k_{0} d_{2} u_{1}\right) & \cdots & \exp \left(\mathrm{j} k_{0} d_{N} u_{1}\right) \\
\exp \left(\mathrm{j} k_{0} d_{1} u_{2}\right) & \exp \left(\mathrm{j} k_{0} d_{2} u_{2}\right) & \cdots & \exp \left(\mathrm{j} k_{0} d_{N} u_{2}\right) \\
\vdots & \vdots & \ddots & \vdots \\
\exp \left(\mathrm{j} k_{0} d_{1} u_{K}\right) & \exp \left(\mathrm{j} k_{0} d_{2} u_{K}\right) & \cdots & \exp \left(\mathrm{j} k_{0} d_{N} u_{K}\right)
\end{array}\right] \in \mathbb{C}^{K \times N}
$$

The main objective here is to calculate multiple excitation vectors from (2). Assume that these excitation vectors of $N$ length are $P(P \ll N)$ sparse, and can be reconstructed accurately from fewer measurements of $\boldsymbol{F}_{R E F}^{(m)}$. That means each vector has only $P$ non-zero entries, thus only $P$ antenna elements need to be excited with feeding currents and can be regarded as "on" state. Therefore, the multiple-pattern synthesis is recast as a simultaneous sparse approximation problem and solved by searching out the sparsest excitation vectors. The above design problem can be mathematically formulated as

$$
\begin{gathered}
\min \left\|\boldsymbol{w}^{\prime(m)}\right\|_{0} \quad \text { s.t. } \quad \sum_{m=1}^{M}\left\|\boldsymbol{F}_{R E F}^{(m)}-\boldsymbol{F}^{(m)}\right\|_{2} \leq \varepsilon \\
\boldsymbol{F}_{R E F}^{(m)}=\boldsymbol{\Phi} \boldsymbol{w}^{(m)}+\boldsymbol{e}
\end{gathered}
$$

where $\boldsymbol{F}_{R E F}^{(m)}=\left[F_{R E F}^{(m)}\left(u_{1}\right), F_{R E F}^{(m)}\left(u_{2}\right), \ldots, F_{R E F}^{(m)}\left(u_{K}\right)\right]^{\mathrm{T}} \in \mathbb{C}^{K}$ is a sample vector of the $m$ th reference pattern at different $K$ observation angle. $\boldsymbol{e}=\left[e_{1}, e_{2}, \ldots, e_{K}\right]^{\mathrm{T}} \in \mathbb{C}^{K}$ is a zero-mean complex Gaussian error vector with user-defined variance $\sigma^{2}$ proportional to $\varepsilon$, and $\varepsilon$ is the maximum matching error.

If the real part and imaginary part of $\boldsymbol{w}^{(m)}$ are represented as two real weight vectors $\boldsymbol{w}_{R}^{(m)}$ and $\boldsymbol{w}_{I}^{(m)}$, the sparse matrix expression (5) can be expanded according to the real part and imaginary part as follows

$$
\left[\begin{array}{c}
\operatorname{Re}\left(\boldsymbol{F}_{R E F}^{(m)}\right) \\
\operatorname{Im}\left(\boldsymbol{F}_{R E F}^{(m)}\right)
\end{array}\right]=\left[\begin{array}{c}
\operatorname{Re}(\boldsymbol{\Phi}) \\
\operatorname{Im}(\boldsymbol{\Phi})
\end{array}\right] \boldsymbol{w}_{R}^{(m)}+\left[\begin{array}{c}
-\operatorname{Im}(\boldsymbol{\Phi}) \\
\operatorname{Re}(\boldsymbol{\Phi})
\end{array}\right] \boldsymbol{w}_{I}^{(m)}+\left[\begin{array}{c}
\operatorname{Re}(\boldsymbol{e}) \\
\operatorname{Im}(\boldsymbol{e})
\end{array}\right]
$$

Set $\overline{\boldsymbol{w}}^{(m)}=\left[\boldsymbol{w}_{R}^{(m)}, \boldsymbol{w}_{I}^{(m)}\right]^{\mathrm{T}} \in \mathbb{R}^{2 N}, \overline{\boldsymbol{F}}_{R E F}^{(m)}=\left[\operatorname{Re}\left(\boldsymbol{F}_{R E F}^{(m)}\right), \operatorname{Im}\left(\boldsymbol{F}_{R E F}^{(m)}\right)\right]^{\mathrm{T}} \in \mathbb{R}^{2 K}, \overline{\boldsymbol{e}}=[\operatorname{Re}(\boldsymbol{e}), \operatorname{Im}(\boldsymbol{e})]^{\mathrm{T}} \in \mathbb{R}^{2 K}$, and

$$
\overline{\boldsymbol{\Phi}}=\left[\begin{array}{cc}
\operatorname{Re}(\boldsymbol{\Phi}) & -\operatorname{Im}(\boldsymbol{\Phi}) \\
\operatorname{Im}(\boldsymbol{\Phi}) & \operatorname{Re}(\boldsymbol{\Phi})
\end{array}\right]
$$

The expression (6) can be written as 


$$
\overline{\boldsymbol{F}}_{R E F}^{(m)}=\overline{\boldsymbol{\Phi}} \overline{\boldsymbol{w}}^{(m)}+\overline{\boldsymbol{e}}
$$

Therefore, the sparse weight vectors can be calculated by

$$
\boldsymbol{w}^{\prime(m)}=\bar{w}_{n}^{(m)}+\mathrm{j} \bar{w}_{n+N}^{(m)} \quad n=1, \ldots, N
$$

It is known from (9) that each element of $\overline{\boldsymbol{w}}^{(m)}$ is treated as statistically independent. This will lead to the array weights are often either purely real or purely imaginary, neglecting that sparse complex layouts frequently exhibit non-negligible real and imaginary components at the same element positions. Therefore, the real part $\boldsymbol{w}_{R}^{(m)}$ and imaginary part $\boldsymbol{w}_{I}^{(m)}$ should be respectively solved, and (5) can be equivalent to

$$
\begin{aligned}
\tilde{\boldsymbol{F}}_{R}^{(m)} & =\tilde{\boldsymbol{\Phi}}_{R} \boldsymbol{w}_{R}^{(m)}+\tilde{\boldsymbol{e}}_{R} \\
\tilde{\boldsymbol{F}}_{I}^{(m)} & =\tilde{\boldsymbol{\Phi}}_{I} \boldsymbol{w}_{I}^{(m)}+\tilde{\boldsymbol{e}}_{I}
\end{aligned}
$$

where $\tilde{\boldsymbol{\Phi}}_{R}=[\operatorname{Re}(\boldsymbol{\Phi}), \operatorname{Im}(\boldsymbol{\Phi})]^{\mathrm{T}} \in \mathbb{R}^{2 K \times N}$ and $\tilde{\boldsymbol{\Phi}}_{I}=[-\operatorname{Im}(\boldsymbol{\Phi}), \operatorname{Re}(\boldsymbol{\Phi})]^{\mathrm{T}} \in \mathbb{R}^{2 K \times N}$ are two new observation matrixes. $\tilde{\boldsymbol{F}}_{R}^{(m)} \in \mathbb{R}^{2 K}$ and $\tilde{\boldsymbol{F}}_{I}^{(m)} \in \mathbb{R}^{2 K}$ are the real vectors, moreover, they satisfy the condition $\tilde{\boldsymbol{F}}_{R}^{(m)}+\tilde{\boldsymbol{F}}_{I}^{(m)}=\overline{\boldsymbol{F}}_{R E F}^{(m)}$. $\tilde{\boldsymbol{e}}_{R} \in \mathbb{R}^{2 K}$ and $\tilde{\boldsymbol{e}}_{I} \in \mathbb{R}^{2 K}$ are two zero-mean complex Gaussian error vectors with variance $\sigma^{2} / 2$, moreover, they satisfy the condition $\tilde{\boldsymbol{e}}_{R}+\tilde{\boldsymbol{e}}_{I}=\overline{\boldsymbol{e}}$. Therefore, the sparse weight vectors can be calculated by

$$
\boldsymbol{w}^{(m)}=\boldsymbol{w}_{R}^{(m)}+\mathbf{j} \boldsymbol{w}_{I}^{(m)}
$$

In summary, the optimal design of sparse reconfigurable arrays is recast as a simultaneous sparse approximation problem, and a novel method is proposed to solve this problem. In light of the multiple measurement vector (MMV) model, statistical correlation of different tasks can be exploited to enhance the performance of simultaneous inversion. Then multiple response extension of the sparse Bayesian learning (SBL) named M-SBL can be used to solve this model. The sparse weight vectors with the same zero elements can be effectively estimated once the corresponding observation matrixes and measurement vectors are known. In the framework of M-SBL, the corresponding sparse representation can be expressed as

$$
\underline{\boldsymbol{F}}=\underline{\boldsymbol{\Phi}} \underline{\boldsymbol{W}}+\underline{\boldsymbol{e}}
$$

Once sparseness prior on $\boldsymbol{W}$ is known, the original synthesis problem can be converted into a Bayesian linear regression problem. The estimate of this sparse weight vector can be searched out with a maximum a posteriori (MAP) estimator. Then the posterior probability density function over $\underline{W}$ turns out to be

$$
p(\underline{\boldsymbol{W}} \mid \boldsymbol{r}, \underline{\boldsymbol{F}})=\mathrm{N}(\boldsymbol{\Gamma}, \boldsymbol{\Sigma})
$$

where $\boldsymbol{r}=\left[r_{1}, r_{2}, \ldots, r_{N}\right]^{\mathrm{T}}$ is a shared hyper-parameter vector, $\boldsymbol{\Gamma}$ and $\boldsymbol{\Sigma}$ are the posterior mean and covariance respectively

$$
\Gamma=\left[\mu^{(1)}, \ldots, \mu^{(T)}\right]=\Lambda^{1 / 2}\left(\underline{\Phi} \Lambda^{1 / 2}\right)^{+} \underline{F} \quad \Sigma=\Lambda-\Lambda^{3 / 2}\left(\underline{\Phi} \Lambda^{1 / 2}\right)^{+} \underline{\Phi}
$$

where $\boldsymbol{\Lambda}=\operatorname{diag}\left(r_{1}, r_{2, \ldots}, r_{N}\right)$ is a diagonal matrix whose diagonal element is the corresponding entry of $\boldsymbol{r}$. By maximizing the logarithm of posterior probability density function, we have the following expression to reestimate $\boldsymbol{r}$ and $\sigma^{2}$

$$
\begin{gathered}
\tilde{r}_{n}=\frac{\left\|\boldsymbol{\mu}^{(t)}\right\|_{2}^{2}}{T\left(1-r_{n} \sum_{n n}\right)} \\
\tilde{\sigma}^{2}=\frac{\|\underline{\boldsymbol{F}}-\underline{\boldsymbol{\Phi}} \boldsymbol{\Gamma}\|_{2}^{2}}{T\left[K-N+\sum_{n=1}^{N}\left(\sum_{n n} / r_{n}\right)\right]}
\end{gathered}
$$

where $\sum_{n n}$ is the nth diagonal element of $\Sigma$. By iterating the above two expression and (14) until a convergence criterion has been satisfied, the estimated value of the sparse weight vector can be written as

$$
\underline{\hat{\boldsymbol{W}}}=\underset{\underline{\hat{\boldsymbol{W}}}}{\arg \max }\left\{\left.p(\underline{\boldsymbol{W}} \mid \boldsymbol{r}, \underline{\boldsymbol{F}})\right|_{r=\hat{r}}\right\}=\left.\hat{\Gamma}\right|_{\boldsymbol{r}=\hat{\boldsymbol{r}}}
$$

To sum up, the optimal design of sparse configurable arrays is recast as a MMV model in this paper, and the M-SBL method is exploited to solve the sparsest weight vectors.

\section{Numerical Analysis and Assessment}


Based on the MMV model of multiple patterns, a representative experiment is devoted to validate the effectiveness and advantages of the proposed method. We take as reference two different patterns, which consists of a Dolph-Chebyshev pencil beam with PSL=-20dB, and a flat-top beam synthesized by Woodward-Lawson method. These patterns are generated with 30 isotropic $\lambda / 2$-spaced antenna elements. The comparison of the reference patterns and the achieved pattern synthesized by the M-SBL method is shown in Fig. 1. It is observed that the proposed method achieves a perfect reconstruction of the desired patterns. Fig. 2 shows the element positions and excitations synthesized by the reference array and the obtained sparse array. As can be seen, only 20 antenna elements are required for this obtained sparse array, and the element saving with respect to the uniform array is $1 / 3$.

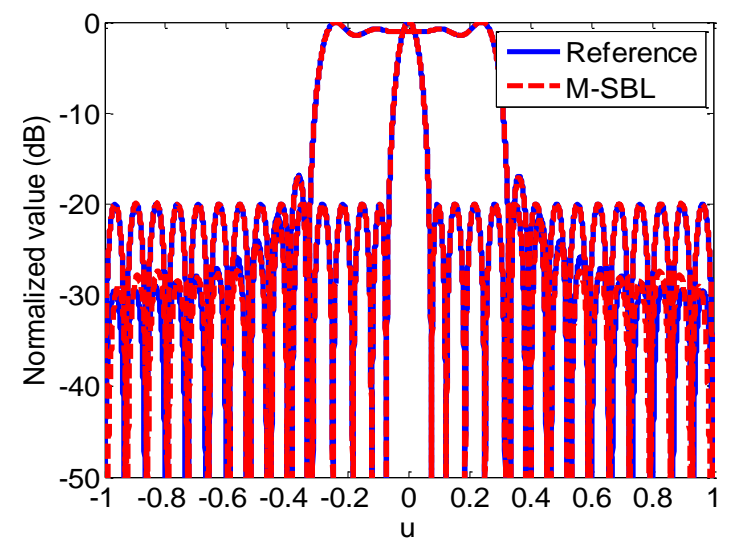

Fig. 1: Power patterns synthesized by the reference array and the M-SBL method.

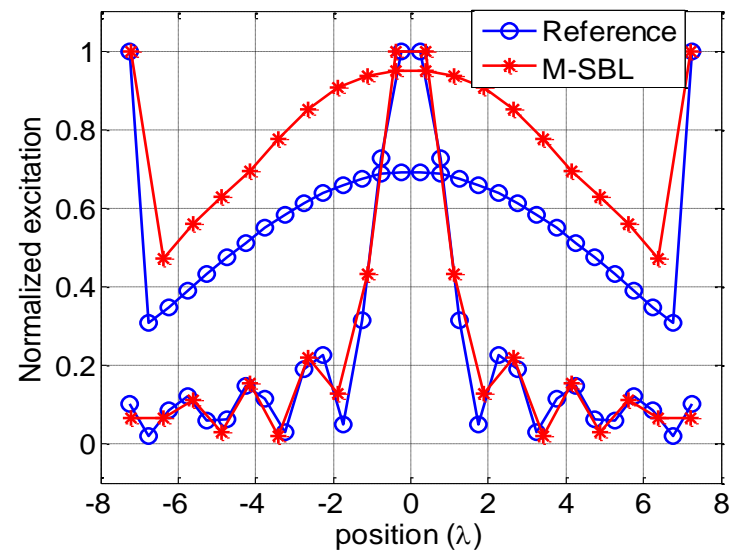

Fig. 2: Element positions and excitations of the reference array and the M-SBL method.

\section{Conclusions}

In this paper, the M-SBL method is proposed for the optimal design of sparse reconfigurable arrays. The synthesis of sparse arrays is recast as a simultaneous sparse approximation problem and modeled with multiple measurement vector. Then the M-SBL method is exploited to solve the above optimization problem. The common element positions and individual element excitations for multiple radiation patterns are achieved simultaneously by searching out multiple sparse weight vectors. Through varying excitation distributions, different radiated patterns are generated by a sparse reconfigurable array, in which the number of antenna elements is saved by $1 / 3$ while providing an excellent pattern matching. A representative numerical experiment has been presented to prove the effectiveness of the proposed method.

\section{Acknowledgements}

This work was supported by the National Natural Science Foundation of China under Grant 61172148 and 61671465 . The authors would like to thank the associate editor and the anonymous reviewers for their helpful comments and suggestions. 


\section{References}

[1] R. L. Haupt, M. Lanagan. Reconfigurable antennas. IEEE Antennas and Propagation Magazine, 2013, 55, (1): 4961

[2] B. Z. Wang, S. Q. Xiao, Y. Y. Bai, G. M. Zhang. Researches on pattern reconfigurable antenna and its application in phase array. Proceeding of the International Workshop on Antenna Technology, HongKong, China, 2011: 46-49.

[3] G. K. Mahanti, S. Das, A. Chakraborty. Design of Phase-Differentiated Reconfigurable Array Antennas With Minimum Dynamic Range Ratio. IEEE antennas and propagation letters, 2006, 5: 262-264.

[4] W. Kang and K. Kim. A radiation pattern-reconfigurable antenna for wireless communications. IEEE International Symposium on Antennas and Propagation (APSURSI), 2011, 1545-1548.

[5] D. Gies, Y. Rahmat-samii. Particle swarm optimization for reconfigurable phase differentiated array design," Microw. Opt. Technol. Lett, 2003, 38: 168-175.

[6] G. K. Mahanti, A. Chakraborty, S.Das. Design of fully digital controlled reconfigurable array antennas with fixed dynamic range ratio. Journal of Electromagnetic Waves and Applications, 2007, 21 (1): 97-106.

[7] R. Vescovo. Reconfigurability and beam scanning with phase-only control for antenna arrays. IEEE Transactions on Antenna and Propagation, 2008, 56(6) 1555-1565.

[8] M. Durr, A. Trastoy, and F. Ares. Multiple-pattern linear antenna arrays with single prefixed amplitude distributions: modified Woodward- Lawson synthesis. Electronics Letters. 2000, 36(16): 1345-1346.

[9] W. P. Wipf and B. D. Rao. An empirical Bayesian strategy for solving the simultaneous sparse approximation problem. 2007, 55(7): 3702-3716. 